\title{
Is it time to reconsider the administration of thiamine alone or in combination with vitamin $C$ in critically ill patients? A meta-analysis of clinical trial studies
}

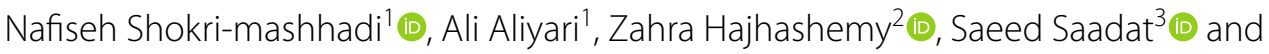 \\ Mohammad Hossein Rouhani $2^{*}$ (1)
}

\begin{abstract}
Background: Although the effect of thiamine alone or in combination with vitamin C has been studied in multiple trials (RCT and interventional studies), their results are inconsistent. This meta-analysis aimed to assess impact of thiamine administration alone, thiamine in combination with vitamin C, and co-administration of low-dose hydrocortisone, vitamin C and thiamine (HVT) on clinical outcomes in critically ill patients.

Methods and materials: After electronic searches on PubMed, Scopus, Cochrane Library, and Web of Science databases, initially 3367 papers were found, and 20 interventional studies were included in our analysis. We assessed the risk-difference between treatment and control (standard treatment) groups by pooling available data on ICU length of stay, number of ventilator free days, mortality, and changes in Sequential Organ Failure Assessment (SOFA) scores.

Results: The results of present studies revealed no significant effect of thiamine in combination with vitamin C, and HVT on number of free days of ventilation. Thiamine alone supplementation was associated with high mortality percentage (WMD: $5.17 \%$; $95 \% \mathrm{Cl}: 2.67,7.67)$. Thiamine in combination with vitamin C had no significant impact on mortality rate. In contrast, HVT could decrease mortality rate (WMD: - 7.23\%; 95\% Cl: - 10.31, - 4.16; I-square: 0.0\%). There was no significant effect of thiamine alone, co-administration of thiamine and vitamin C, and HVT on ICU length of stay. The results of the meta-analysis showed that thiamine alone and HVT supplementation had no significant effect on SOFA score. Interestingly, co-supplementation of thiamine and vitamin $\mathrm{C}$ had a significant decreasing effect on SOFA score (WMD: $-0.73 ; 95 \% \mathrm{Cl}:-1.29,-0.17$; I-square: $0.0 \%)$.
\end{abstract}

Conclusion: In contrast to HVT, thiamine supplementation alone was associated with increased mortality rate in ICU. However, co-supplementation of thiamine and vitamin C had a significant decreasing effect on SOFA score.

\footnotetext{
*Correspondence: sm_rouhani@nutr.mui.ac.ir; s_m_rouhani2003@yahoo.

com

${ }^{2}$ Food Security Research Center and Department of Community

Nutrition, School of Nutrition and Food Science, Isfahan University of Medical Sciences, Isfahan, Iran

Full list of author information is available at the end of the article
}

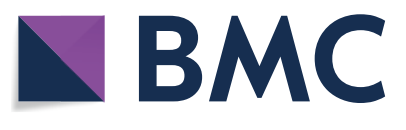

(c) The Author(s) 2022. Open Access This article is licensed under a Creative Commons Attribution 4.0 International License, which permits use, sharing, adaptation, distribution and reproduction in any medium or format, as long as you give appropriate credit to the original author(s) and the source, provide a link to the Creative Commons licence, and indicate if changes were made. The images or other third party material in this article are included in the article's Creative Commons licence, unless indicated otherwise in a credit line to the material. If material is not included in the article's Creative Commons licence and your intended use is not permitted by statutory regulation or exceeds the permitted use, you will need to obtain permission directly from the copyright holder. To view a copy of this licence, visit http://creativecommons.org/licenses/by/4.0/. The Creative Commons Public Domain Dedication waiver (http://creativeco mmons.org/publicdomain/zero/1.0/) applies to the data made available in this article, unless otherwise stated in a credit line to the data. 


\section{Highlights}

- Thiamine, co-supplementation of thiamine and vitamin $\mathrm{C}$ and co-administration of hydrocortisone, vitamin $\mathrm{C}$ and thiamine (HVT) had no significant effect on free days of ventilation and ICU length of stay.

- HVT could decrease and thiamine supplementation could increase mortality rate in ICU.

- Co-supplementation of thiamine and vitamin C could decrease SOFA score.

Keywords: Intensive care unit, Thiamine, Vitamin C, Low-dose hydrocortisone, Meta-analysis

\section{Introduction}

Evidence supports the essential role of thiamine, a watersoluble vitamin, as an essential cofactor in maintenance of normal metabolism and central nervous function [1, 2]. Thiamine deficiency can lead to severe complications, including elevated lactate levels, cardiac failure, and brain dysfunction, which are linked to poor clinical outcomes and finally result in organ failure and mortality [3, 4].

It is reported that circulating thiamine levels in critically ill patients decrease over time, in particular in patients with diabetic ketoacidosis, chronic diarrhea, receiving chronic diuretic therapy, and the predominantly carbohydrate-based diet $[5,6]$. Moreover, malnutrition is a common difficulty in the majority of patients admitted to hospital, especially in the intensive care unit (ICU), that could be potentially associated with an insufficient vital nutrients intake $[7,8]$. In this concern, recent trials have focused on the efficacy of thiamine administration alone or in the combination with vitamin $\mathrm{C}$ in critically ill patients to achieve the best clinical outcomes for these patients. The results of some studies indicated that administration of thiamine alone could improve oxygen consumption in cardiac surgery patients [9], and be effective for prevention of postoperative delirium [10]. Moreover, it has beneficial effects in patients with burns and multiple trauma [11]. Nevertheless, the beneficial impact of thiamine supplementation did not confirm in the other studies [12, 13]. In this regard, previous published systematic review and meta-analysis investigated the effectiveness of vitamin $\mathrm{C}$, thiamine, or co-administration of low-dose hydrocortisone, vitamin $\mathrm{C}$ and thiamine (HVT) on clinical outcomes in critically ill patients. However, the impact of treatment with thiamine alone has been not considered in mentioned studies [14-16]. Furthermore, there were no subgroup analyses based on the use of lowdose hydrocortisone or placebo in the control groups that it can affect the quality of results $[14,15]$. It seems that pooled subgroup analyses from trials focused on administration of thiamine, may help clear information around thiamine supplementation for critically ill patients [17].

The main purpose of this review was to assess the effect of thiamine, co-supplementation of thiamine and vitamin
C, and HVT on the clinical outcomes of adult critically ill patients.

\section{Materials and methods Literature research and data extraction}

Current systematic review was carried out in compliance with the PRISMA statement [18]. Two investigators (N.Sh-m and A.Aliyari) independently conducted an electronic literature search using PubMed, Scopus, Cochrane Library, and Web of Science databases without any restrictions on language or date to identify the effect of thiamine alone or in conjunction with vitamin $\mathrm{C}$ and/ or low-dose hydrocortisone when compared to standard care or placebo (From inception to November 2020). The electronic search strategy was done using the following keywords: ("Critical Care" OR "Critical Illness" OR "critical ill" OR "Intensive Care Units" OR "intensive care") AND (thiamin* OR thiamine OR vitamin B1). The references pointed in the retrieved articles were also searched manually. In addition, similar queries were used for controlled vocabulary search [Mesh] at the same time.

All studies, which assessed the effect of administration of thiamine alone or in combination with vitamin $\mathrm{C}$ or/ and low-dose hydrocortisone versus non-thiamine on patients admitted to ICU were included in this review. The exclusion criteria were as follows: (1) duplicated publications; (2) pediatric studies; (3) observational studies; (4) studies involving other supplements or treatments; (5) lacking the data of predefined endpoints; (6) animal models or in vivo studies; (7) review and meta-analysis studies, chapter's book, and conference papers; and (8) retrospective clinical trial. Patient population consisted of critically-ill adult patients ( $\geq 18$ years). Following outcomes were considered in the present study: (1) SOFA score: It is a simple and objective score that allows for calculation of both the number and the severity of organ dysfunction in six different organs (respiratory, coagulatory, liver, cardiovascular, renal, and neurologic) [19]; (2) Length of stay in the ICU: It is a term introduced by the National Health Service (NHS) as the length of an inpatient hospitalization in ICU, calculated from the day when patients were admitted to the ICU to day of 
discharge based on the number of overnight stays [20]; (3) Ventilator-free days: Ventilator-free days at 28 days is an outcome that depends on organ dysfunction. It is defined in a 28 day period in critically ill patients and measured by the number of free days from mechanical ventilation to evaluate the efficacy of interventions [21]; and Mortality. Following the assessment of titles and abstracts, all identified studies were acquired as full-text. A third reviewer resolved any disagreements by discussion (Z.H).

We extracted the following information: author's name, publication year, participant characteristics (sample size, gender, and age), medical conditions, intervention (type of compounds, dose, and duration) and main outcomes, including mortality, Sequential Organ Failure Assessment (SOFA) score, ICU length of stay, number of ventilator free days, and ICU mortality. The data were extracted independently by two investigators. We also contacted the corresponding author to obtain the data when necessary (N.Sh-M).

\section{Assessment of bias}

In current meta-analysis, the quality assessment of included studies was performed through the use of Cochrane quality assessment tool [22]. Considering the Cochrane checklist, risk of bias for each study was examine based on 7 items including random sequence generation, allocation concealment, reporting bias, performance bias, detection bias, attrition bias, and other sources of bias. Each item was given "high risk" (O), "low risk" ( $\odot$ ) or "unclear risk" (*) score. "High risk" for studies with methodological defect that might affect their results; "low risk" for studies with no methodological defect in that item; and "unclear risk" for studies with insufficient information to examine the impact of method on findings. We considered the overall risk of bias for an article: low for studies that obtained "low risk" score in all domains. Moderate for studies with one or more "unclear risk" score, and high for studies with one or more "high risk" score (Table 1).

\section{Statistical analysis}

The effect of thiamine alone, co-supplementation of thiamine and vitamin $\mathrm{C}$ and/or HVT on clinical outcomes were evaluated by assessing the risk-difference between treatment and control (standard treatment) groups by pooling available data on mortality, ICU length of stay, change of SOFA score, and number of ventilator free days. Values are expressed as mean $\pm S D$. In the cases that the risk-difference between treatment and control was reported as standard error (SE), we converted values to standard deviation (SD) using the formula $\mathrm{SD}=\mathrm{SE} \times \sqrt{ } \mathrm{N}$. Mortality was reported as percent change in included studies. All outcomes were reported in a same values through the studies. The weighted mean difference was calculated by pooling effect sizes via a random-effects mode. Cutoffs for confidence intervals and definitions for both statistical significance $(p<0.05)$ and between-study heterogeneity $\left(I^{2}>50 \%\right)$ was considered. Robustness of the overall effect sizes was tested by sensitivity analysis Begg's rank correlation test and Egger's linear regression test were run to evaluate publication bias. Statistical analyses were performed using Stata software (version 11.2, Stata Corporation, College Station, Texas, USA).

\section{Results}

\section{Study characteristics}

According to the search strategy, 3367 papers were initially found. After removing 1849 duplicates and 1478 unappropriated topic papers, 1518 studies were screened by title and abstract. The remaining 40 studies were considered to be of relevance, and full papers were carefully screened. In the following, 1 conference paper, 2 study protocol, 1 case-report study, 1 inappropriate statistical analysis, and 15 having no specified mentioned data were rejected. Therefore, 20 articles were included in the systematic review. Finally, we excluded 5 studies due to retrospective clinical trial design, and just 15 clinical trial articles enrolled in the meta-analysis [10, 12, 13, 23-34]. The selection process of papers summarized in Fig. 1.

Among 20 articles, fifteen studies were done in single center, and two trials conducted in two centers. Studies were done in different countries, including the USA [1, $8,9,11,12,23]$, Australia [14, 16], Korea [17, 18], Iran $[2,7]$, India [4, 10], Japan [3], China [5], Indonesia [13], Malaysia [15], and Thailand [22]. In addition, one of them has simultaneously done in Brazil, Australia, and New Zealand [6]. Fourteen studies performed on patients with same condition (sepsis), one study on patients with Acute respiratory distress syndrome (ARDS), one study on patients with cardiac arrest condition, and four studies on patients undergoing surgery (coronary artery bypass cardiac, and gastrointestinal surgery). Approximately 58\% of all patients were male. The mean ages of participants ranged from 54 to 72 years, but one study recruiting patients with a mean age of 42 years [34]. Thiamine was administered in various dosing in the studies, ranging from $0.2 \mathrm{~g}$ (g) daily to 0.6 . The dosage of administration of vitamin $\mathrm{C}$ with thiamine was ranged from $2 \mathrm{~g}$ /day to 6 , and the duration of supplementation was various from 2 to 10 days. However, Luger et al. conducted an intervention to identify the effect of a single dosage of $0.3 \mathrm{~g}$ intravenous thiamine supplementation directly before surgery [33]. Two studies reported the serum thiamine status of patients before intervention. In addition, two studies recorded the serum levels of vitamin $\mathrm{c}$ in their subjects. 
Table 1 Quality assessment of included studies in the meta-analysis

\begin{tabular}{|c|c|c|c|c|c|c|c|}
\hline & 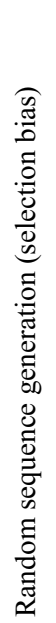 & 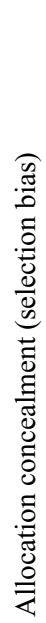 & 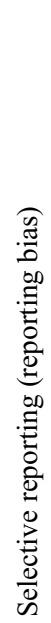 & 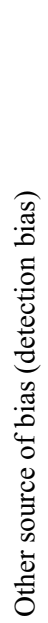 & 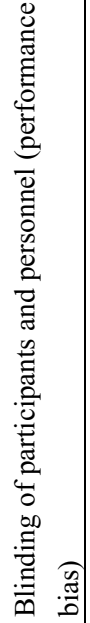 & 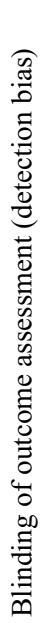 & 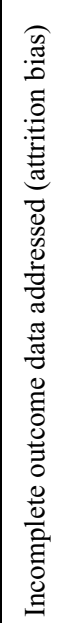 \\
\hline Iglesias, 2020 & & & & & + & & \\
\hline Chang, 2020 & & & & & & & \\
\hline Donnino, 2016 & & & & & & & \\
\hline Balakrishnan, 2019 & & & & & & & \\
\hline Fujii, 2020 & & & & & & & \\
\hline Harun, 2019 & & & & & & & \\
\hline Hwang, 2020 & & & & & & & \\
\hline Karimpour, 2019 & & & & & & & \\
\hline Lomivorotov, 2019 & & & & & & & \\
\hline Luger, 2015 & & & & & & & \\
\hline Moskowitz, 2020 & & & & & & & \\
\hline Moslemi, 2020 & & & & & & & \\
\hline Nasution, 2020 & & & & & & & \\
\hline Pradita-ukrit, 2020 & & & & & & & \\
\hline Wani, 2020 & & & & & & & \\
\hline
\end{tabular}




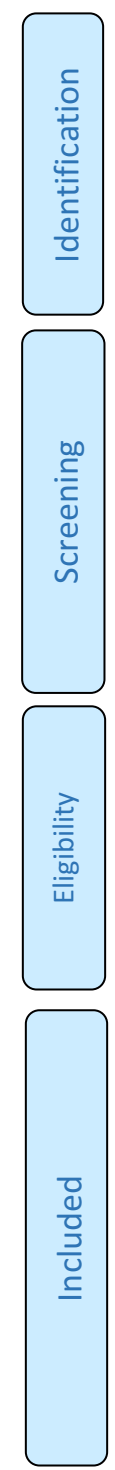

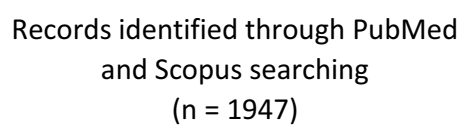

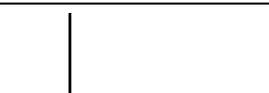

Records after duplicates removed

$$
(n=1518)
$$

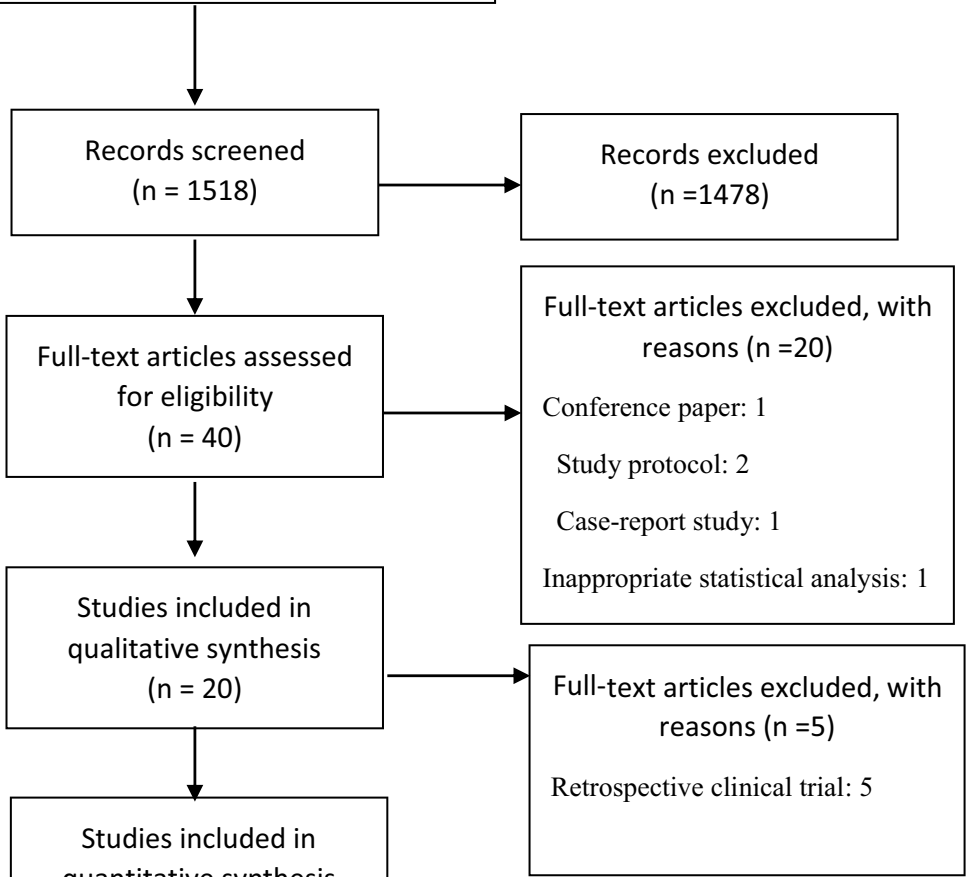

quantitative synthesis

(meta-analysis)

$$
(n=15)
$$

Fig. 1 PRISMA flowchart of study selection process for effect of vitamin B1 on clinical outcomes of ICU patients

After removing retrospective interventions, we enrolled clinical trial articles in the meta-analysis. Characteristics of clinical trial studies have been summarized in Table 2.

\section{Thiamine regimens}

The results of the studies have been displayed in three main subgroups. The first subgroup was thiamine supplementation which involved seven interventions with thiamine injection $[2,9,13-16,22]$. The second subgroup included three studies. Intervention for this subgroup was the concurrent injection of thiamine in association with vitamin $C[7,17,18]$. The last subgroup, which included ten studies, was the simultaneous injection of thiamine, vitamin $\mathrm{C}$, and low-dose hydrocortisone (triple therapy) $[1,3-6,8,10-12,23]$.

\section{The effect of thiamine alone, thiamine in association with vitamin C, and HVT on number of ventilator-free day}

The results of present studies revealed no significant effect of thiamine in combination with vitamin $C$, and HVT on number of free days of ventilation, respectively (WMD: 0.28; 95\% CI: -0.45 , 1.01; I-square: $0.0 \%$ ) and (WMD: - 0.40; 95\% CI: - 2.16, 1.36; I-square was not calculated). There were no enough study for statistical tests regarding effect of thiamine alone on number of free day ventilation (Fig. 2). 


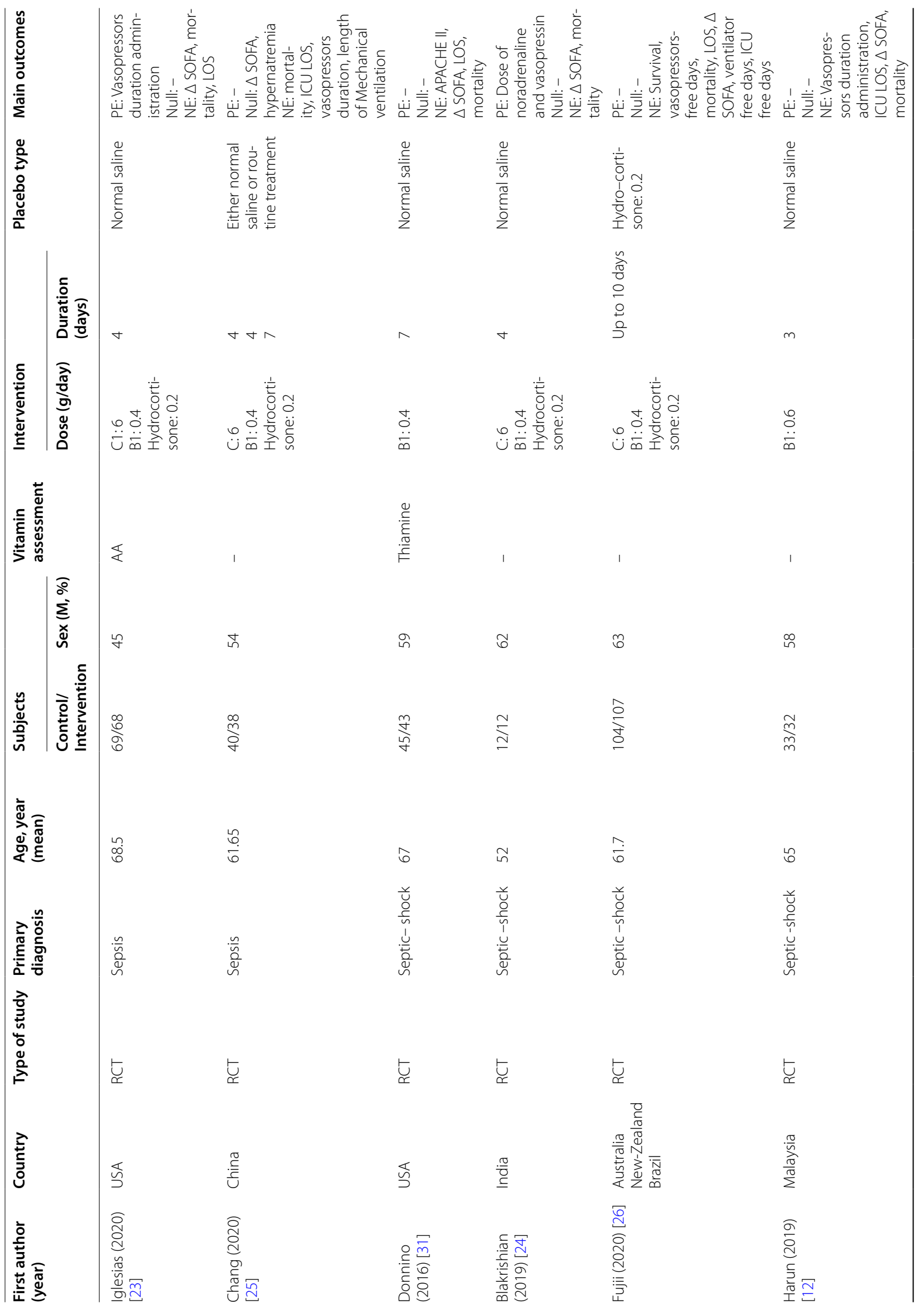




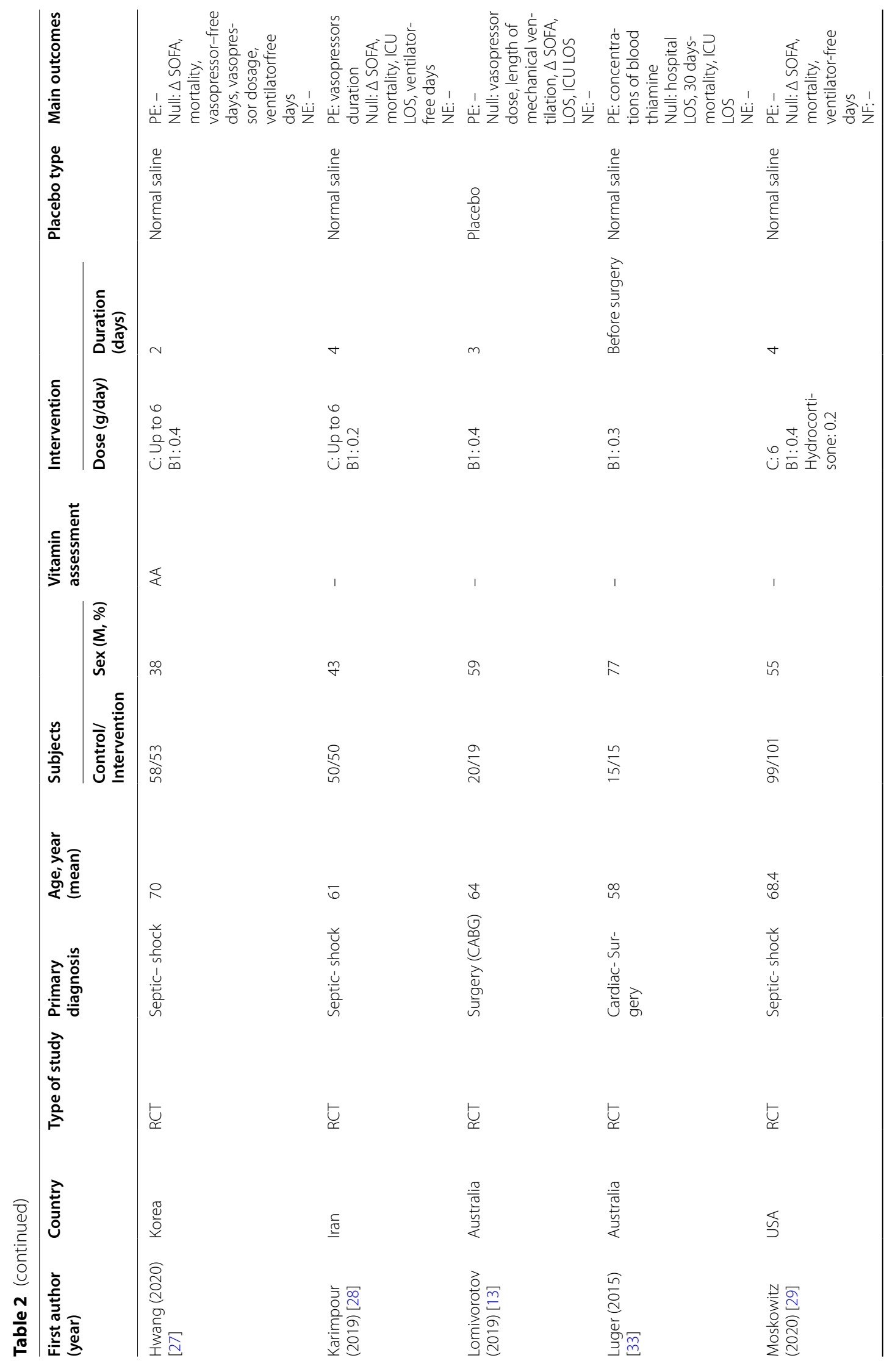




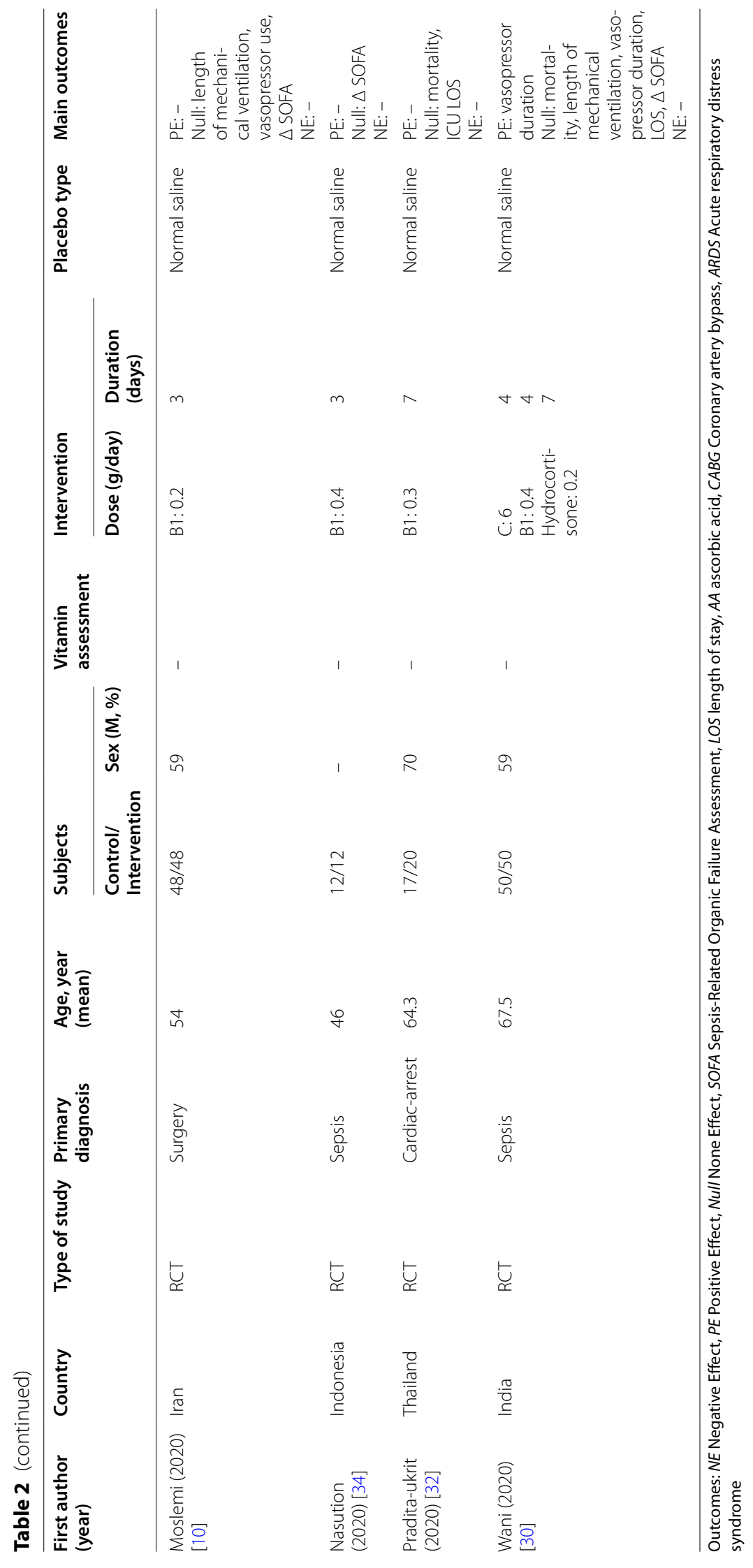




\begin{tabular}{|c|c|c|}
\hline Authors, year & ES $(95 \%$ CI) & Weight \% \\
\hline $\mathbf{B} 1+\mathbf{C}$ & & \\
\hline Fuji & $-1.37(-5.56,2.82)$ & 2.59 \\
\hline Moskowitz & $0.74(-0.51,1.99)$ & 28.99 \\
\hline Hwang & $0.11(-0.81,1.03)$ & 53.76 \\
\hline Subtotal $(I-s q u a r e d=0.0 \%, p=0.537)$ & $0.28(-0.45,1.01)$ & 85.34 \\
\hline B1 + C + Cortisol & & \\
\hline Iglesias & $-0.40(-2.16,1.36)$ & 14.66 \\
\hline Subtotal $(\mathrm{I}$-squared $=. \%, \mathrm{p}=)$. & $-0.40(-2.16,1.36)$ & 14.66 \\
\hline Overall $(\mathrm{I}$-squared $=\mathbf{0 . 0} \%, p=\mathbf{0 . 6 3 1})$ & $0.18(-0.50,0.85)$ & 100.00 \\
\hline NOTE: Weights are from random effects analysis & & \\
\hline-5.56 & 5.56 & \\
\hline
\end{tabular}

The effect of thiamine alone, thiamine in association with vitamin C, and HVT on ICU mortality percentage The effects of different treatments on ICU mortality rate is illustrated in Fig. 3. The results of the meta-analysis of three studies regarding effect of thiamine supplementation on ICU mortality showed that administration of thiamine alone is associated with high mortality percentage without heterogeneity between studies (WMD: 5.17\%; 95\% CI: 2.67, 7.67; I-square: 0.0\%). Thiamine in combination with vitamin $C$ had no significant impact on mortality rate (WMD: $2.19 \%$; $95 \% \mathrm{CI}$ : $-0.88,5.26$; I-square: 95.4\%). In contrast, HVT could decrease mortality rate (WMD: - 7.23\%; 95\% CI: - 10.31, - 4.16; I-square: $0.0 \%)$.

\section{The effect of thiamine alone, thiamine in association with vitamin C, and HVT on length of ICU stay}

The effects of different treatments on length of ICU stay is illustrated in Fig. 4. The meta-analysis of two studies indicated that there was no statistical significant effect of thiamine alone on ICU length of stay (WMD: 0.24; 95\% CI: - 0.72, 1.21; I-square: 41.5\%). Moreover, supplementation of thiamine in association with vitamin $\mathrm{C}$, and HVT have no significant effect on ICU length of stay
(WMD: 0.90; 95\% CI: - 0.17, 1.96; I-square: 11.4\%) and (WMD: 0.08; 95\% CI: - 0.60, 0.76; I-square: 0.0\%), respectively.

The effect of thiamine alone, thiamine in association with vitamin C, and HVT on change of SOFA score The effects of different treatments on SOFA score is illustrated in Fig. 5. The results of the meta-analysis showed that thiamine supplementation had no significant effect on SOFA score in the ICU patients (WMD: 0.13; 95\% CI: $-0.54,0.79$; I-square: $0.0 \%)$. There was no heterogeneity between studies. Interestingly, supplementation with thiamine and vitamin $C$ had a significant decreasing effect on SOFA score (WMD: -0.73 ; 95\% CI: $-1.29,-0.17$; I-square: $0.0 \%)$. There was no heterogeneity the between studies. Additionally, our analysis showed that administration of thiamine, $\mathrm{C}$ and low-dose hydrocortisone had no overall effect on SOFA Score (WMD: -0.39 ; 95\% CI: - 2.61, 1.83; I-square: 95.5\%) with high heterogeneity between studies.

\section{Sensitivity analysis}

The sensitivity analysis regarding the effect of thiamine alone on ICU mortality, the number of ventilator-free 


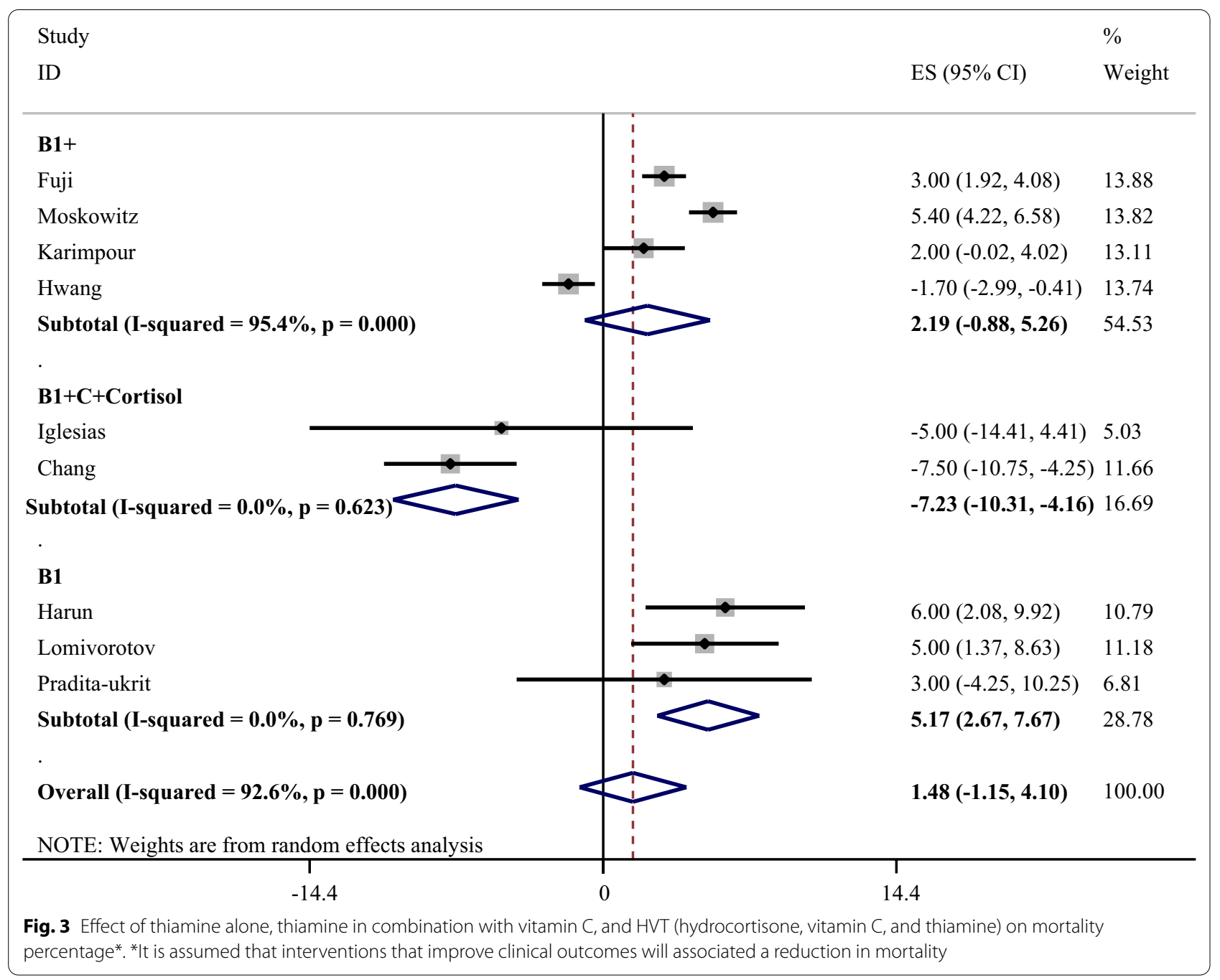

days, and the SOFA score specified that the results were not impressed by any one study. Concerning the effect of thiamine administration alone on length of ICU stay, after excluding an unmatched study Pradita-Ukrit et al.) [32], heterogeneity between studies was disappeared (WMD: $0.61[0.01,1.22]$ ).

Moreover, the results of sensitivity analysis showed that after removing a study conducted by Hwang et al. [27], thiamine supplementation in association with vitamin C has no significant effect on SOFA score (WMD: -0.83 $[-1.97,0.31])$. In contrast, removing the study by Hwang et al. [27] reduced the heterogeneity among the studies. Therefore, the effect of thiamine in association with vitamin $\mathrm{C}$ on length of ICU and ICU mortality becomes significant ((WMD: 2.59 [0.01, 5.18]), (WMD: 3.57 [1.62, $5.51])$ ), respectively. There were no evidences of publication bias for studies regarding effect of thiamine supplementation in combination with vitamin $C$ on number of ventilator-free days.
The sensitivity analysis concerning effect of HVT on number of ventilator free days, and length of ICU stay also revealed no evidences of heterogeneity to be introduced. However, through sensitivity analysis, we found that results of HVT on ICU mortality, and changes of SOFA score was affected by Chang et al. [25], and Balakrishnan et al. [24], respectively [(WMD: -5 [- 14.41, 4.41]), (WMD: -1.44 [- 2.49, - 0.39])].

\section{Discussion}

\section{The effect of thiamine alone on clinical outcomes in ICU patients}

In the current meta-analysis, we identified that administration of thiamine alone had no significant effect on SOFA score, and number of ventilator-free days in ICU patients. In contrast, thiamine supplementation increased mortality percentage in ICU.

Thiamine is an essential cofactor for the synthesis of ATP in mitochondrial and function of antioxidant 


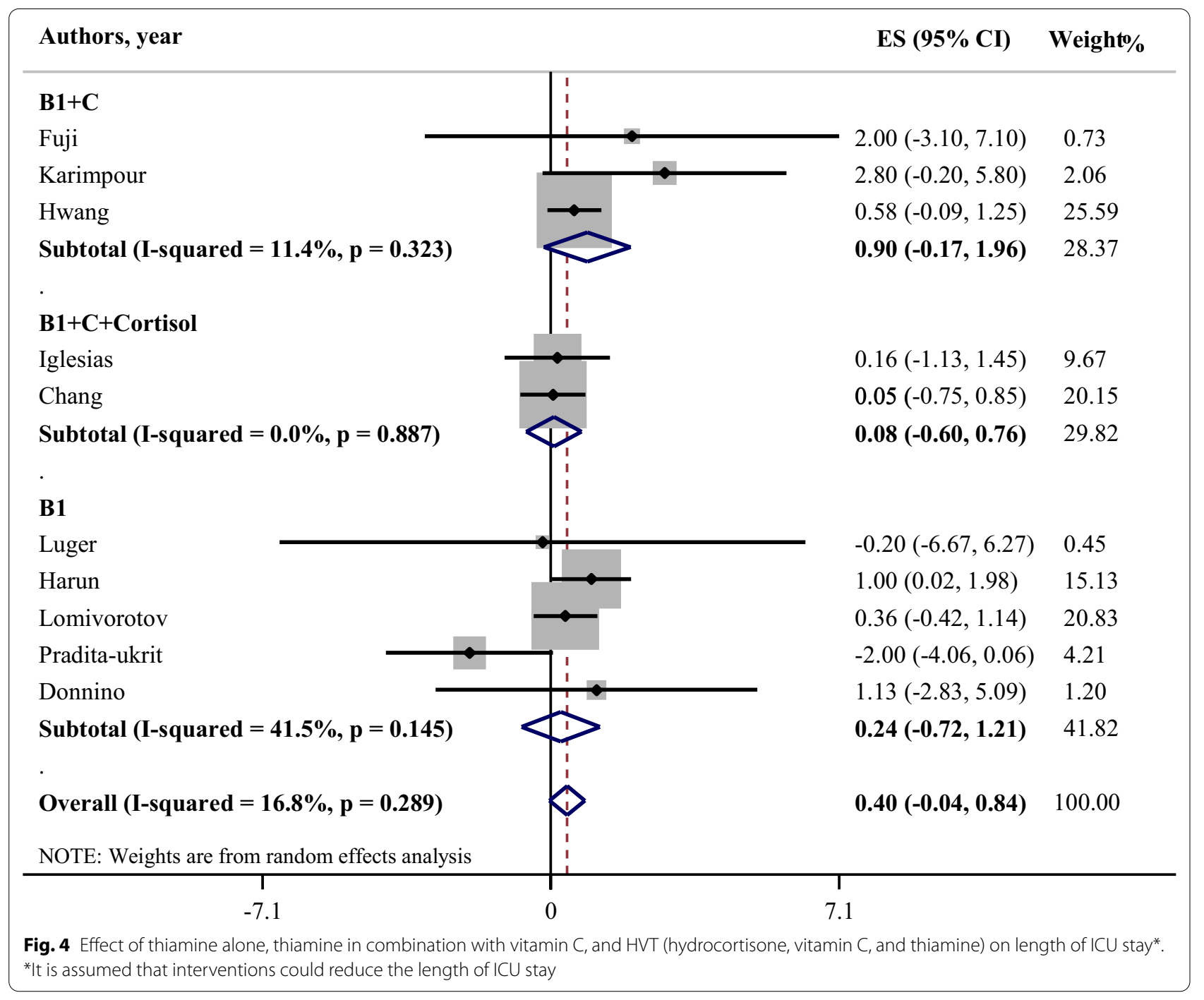

enzymes, as well as it has the critical role in neurological and cardiac function [35, 36]. The hypermetabolic state, administered loop diuretics, and intensive care treatments can lead to thiamine deficiency in critically adult patients $[36,37]$. It seems that the ameliorating effect of thiamine on end-organ damage could be hypothetically associated with the cells' adenosine triphosphate increasing and reduction in lactic acid production, and consequently might have a protective effect on mortality [38]. Unexpectedly, in the current analysis, the increased mortality caused by thiamine administration may only be due to the following reasons. First, it has been previously documented that thiamine administration has no significant effect on lactate clearance in extensive surgery patients [9]. Second, we could not enter some stated positive results related to thiamine treatment on mortality of critically ill patients with septic shock into our analysis, due to inappropriate reported data [39]. Another possible reasons may be related to the status of thiamine deficiency at baseline of intervention, and the median time from admission to thiamine administration in critically ill patients that unfortunately has been not clarified across the included studies [12,17]. It also should be considered that, besides the thiamine deficiency, serum lactate levels could be affected by other factors, including anaerobic glycolysis due to hypoperfusion and microcirculation abnormalities, and impaired hepatic lactate clearance $[40,41]$. Consistent with our study, the findings of a nationwide database-based observational study concerning exanimating the effect of thiamine administration on mortality in 68,571 eligible patients indicated no support for thiamine administration early after admission and the 28-day mortality in patients with septic shock [42]. Moreover, a recent review study by evaluating 122 papers found that although thiamine deficiency may be more prevalent in patients admitted to ICU, 


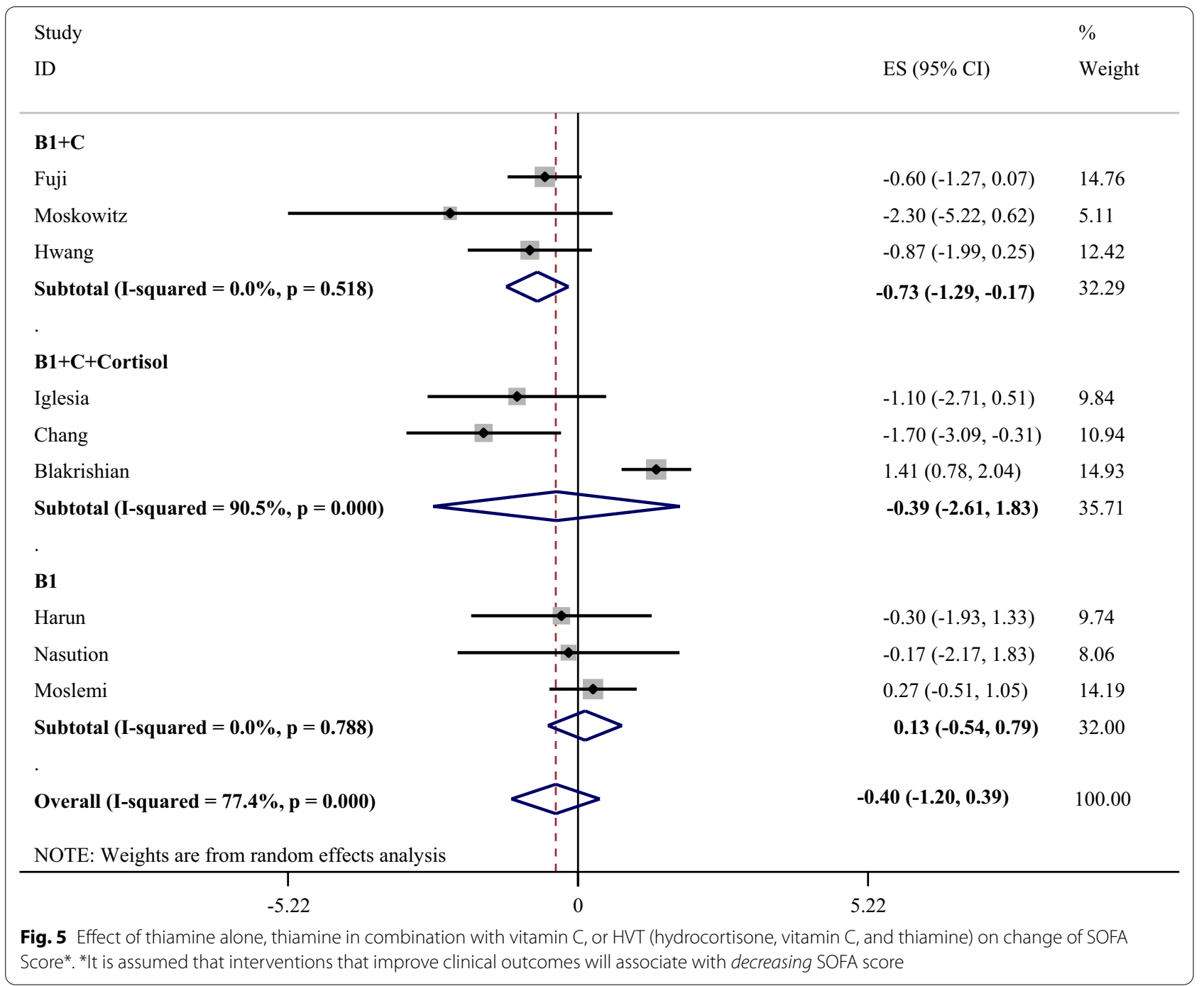

there is a lack of consensus on the benefits of thiamine supplementation in the main outcomes of patients [43]. Therefore, we hypothesized that the positive effect of treatment with thiamine alone on clinical outcomes in critically ill patients could be affected by several factors, including organ function score, the time of thiamine prescription, thiamine deficiency, and lactic acidosis status, particularly in cases with identified nutritional risk factors. Consequently, these results could not be generalized to patients with other conditions, and the potential therapeutic impact of thiamine in the overall ICU population remains unknown.

\section{The effect of thiamine in combination with vitamin C on clinical outcomes in ICU patients}

Our pooled analysis showed that the combination therapy with thiamine and vitamin $\mathrm{C}$ had no significant impact on ICU mortality, number of ventilator free days, and ICU stay. Treatment with thiamine in combination with vitamin $C$ could decrease SOFA score in critically ill patients with sepsis. However, sensitivity analysis revealed that after removing an unmatched study, the mentioned effect on SOFA score has disappeared.

There is evidence that vitamin $C$ levels can decline dramatically as a result of acute inflammation of many physiological stress conditions, such as sepsis, traumas, extensive surgery, and burns [44-46]. As an antioxidant, vitamin $C$ has an important function in cell protection against inflammation and oxidative stressinduced cellular damage [47]. Providing data on circulating antioxidant levels in the largest cohort of septic patients also showed that the capacity of antioxidant serum levels was associated with 30-day survival (hazard ratio $=1.50,95 \%$ confidence interval $=1.16-1.94$, $P=0.002$ ) [48]. In this concern, many studies have examined the potential effect of vitamin $\mathrm{C}$ and thiamine 
on the SOFA score, as the prognostic indicator of mortality in patients with sepsis and post-cardiac arrest syndrome $[49,50]$. In confirmation with our study, Soto et al. also revealed the benefits of antioxidant therapy on multiple organ dysfunction in patients with Coronavirus infection [51]. In contrast, recent published systematic review and meta-analysis concerning impact of vitamin $C$ on patients with sepsis reported that vitamin $C$ supplementation did not have any effect on survival and ICU stay in critically ill patients [52]. However, there are some limitations in mentioned study. First, the information of studies on vitamin $\mathrm{C}$ administration were pooled and analyzed in combination with thiamine to examine the effect of vitamin $\mathrm{C}$ on ICU patients. In addition, the majority of included studies had retrospective design.

Therefore, we assumed that combination therapy with thiamine and vitamin $\mathrm{C}$ might enhance organ function by cell damage reduction, modulation of inflammatory status, and the neurological protection in organs. However, the differences in inflammation status and antioxidant levels of participants could determine other benefits of this combination therapy on ICU outcomes [53]. On the other hand, we showed that improved SOFA score did not lead to a mortality benefit in patients who received thiamine and vitamin $\mathrm{C}$ combination therapy. Although the change in SOFA score has been considered as the primary outcome along with reporting mortality in several studies, some points should be attended in the interpretation of Delta Sofa $(\triangle$ SOFA) score in our study. According our sensitivity analysis, after removing an unmatched study conducted by Hwang et al. [27], the significant impact of thiamine and vitamin $\mathrm{C}$ combination therapy on SOFA score has disappeared. Since the characteristics of study population in mentioned study, including vitamin C deficiency (approximately $50 \%$ of participants) and gender (female, 62\%) might affect the results of our analysis, these statistically significant changes in SOFA score should be cautiously interpreted. Moreover, this outcome was evaluate only in patients who were alive in the ICU on $72-\mathrm{h}$, that means data regarding participants who early discharge from the ICU due to recovery or death were not considered in this analysis. Moreover, through sensitivity analysis we found that our pooled analysis of the changes of SOFA score was affected by Balakrishnan et al. study [24]. For this reason and because of the mentioned variety for measurements and patients' conditions, our finding should be interpreted with caution. Consequently, recommended thiamine and vitamin $C$ dosage may vary according to individual requirements and may need to administrate based on the patients' conditions individually.

\section{The effect of HVT on clinical outcomes in ICU patients}

In the present study, we showed that the HVT protocol could decrease mortality rate. In contrast, HVT had no significant effect on other clinical outcomes.

In the early stage of sepsis, acute organ dysfunction caused by the release of numerous cytokines and metabolic abnormalities emphasizes the important role of early treatment in restoring vascular endothelial function and the reduction of inflammation in these patients [54]. Some previous studies documented that the early treatment with low-dose hydrocortisone in combination with thiamine and vitamin $\mathrm{C}$ could improve organ function and prevent organ injury in patients with septic shock, as indicated by the reduction in SOFA scores $[55,56]$. The findings of recent review also showed that administration of low-dose hydrocortisone, ascorbic acid and thiamine (HAT therapy) may reduce organ dysfunction in patients with sepsis and improved mortality outcomes [57]. Moreover, a recently published meta-analysis by Shi et al., on overall results from cohorts revealed that HVT could significantly reduce mortality (RR0.46, 95\% CI 0.25 to $\left.0.86, p=0.01 ; I^{2}=75 \%, P_{H}=0.001\right)$, but not the duration of vasopressors use (WMD $1.11 \mathrm{~h}, 95 \% \mathrm{CI}-59.60$ to $61.82, p=0.97 ; I^{2}=98 \%, P_{H}<0.001$ ) [58]. However, low dose corticosteroids administration could not effect on short- and longer term mortality in adult's patients with septic shock [59].

Clinically, the correlation between adverse outcomes of respiratory infections and hypertension was indicated and attributed to increased expression of Angiotensinconverting enzyme II (ACE2) [60]. Recently, it has been shown that ascorbic acid administration can play controlling role on cellular expression of ACE2 [61]. On the other hand, administration of low-dose hydrocortisone is recognized as the strongest activator of ACE2 [62]. In addition, the different effects of hydrocortisone on organ function were reported based on dose and time of administration. [63]. It seems that the addition of thiamine and vitamin $\mathrm{C}$, as antioxidants, modulate the effects of hydrocortisone therapy, and may facilitate the binding of glucocorticoids to their receptor [64]. Moreover, impairment of glycemic control, as an important side effect during steroid administration may moderate through regulation of glucose metabolism by thiamine therapy [65]. These results confirm the differences in the effects of intervention between patients at the different stages of sepsis and primary diagnosis condition.

However, in contrast to positive effect of thiamine and vitamin $C$ supplementation on the SOFA score, HVT therapy had no significant improvement in the change of SOFA score in our study. One of the possible reason for this result might be that the SOFA scores calculated at different intervals times in three 
included studies, SOFA measurement intervals at first $72 \mathrm{~h}[23,25]$ versus $96 \mathrm{~h}$ [24]. In addition, the type of disease diagnosed in hospitalized patients, including sepsis and surgery, might make a difference in the outcomes. The recent results of treatment with vitamin $\mathrm{C}$, thiamine, and hydrocortisone indicated a significant reduction in the SOFA score in the intervention group in comparison to the control group in patients with sepsis [68]. Therefore, we speculate that the results of this combination treatment through antioxidant, anti-inflammatory, and immune-enhancing properties depend on patients' conditions. The lack of adequate data in this area suggests more randomized clinical trials regarding the effects of combination treatment on clinical outcomes in ICU patients with various primary conditions, such as surgery or trauma.

\section{Limitations and strengths}

Recently, several meta-analysis studies determined the effect of vitamin $C$ with or without thiamine treatment and reassessed the value of HVT administration in patients with sepsis [66-68]. Strengths of the current systematic review, including the use of a comprehensive database evaluating and consideration of several recently published studies, and the examination of the value of thiamine administration in three main subgroups (thiamine administration individually, thiamin in combination with vitamin $\mathrm{C}$, and HVT through 15 clinical studies that can improve our information concerning the management of clinical outcomes in critically ill patients. This meta-analysis, nonetheless, has potential limitations that should be considered. First, as stated, the pooled effect of thiamine alone or in combination with vitamin $\mathrm{C}$ was evaluated only on patients with sepsis or under surgery due to data deficiencies. Second, since the results related to mortality in the eligible studies was reported as percent changes, we typically could not identify risk difference. In addition, due to the limited reporting on the topic of impact of thiamine administration alone and HVT, we consequently could not analyze and determine the effect of thiamine administration on number of ventilation free day. In addition, because of limited data, we could not evaluate the heterogeneity regarding the large variability of SOFA measurement intervals by subgroup analysis. The heterogeneity of background conditions of patients from sepsis to trauma also is another limitation highlighting the need for more randomized clinical trials regarding the effects of combination treatment on clinical outcomes with various primary conditions of patients admitted to ICU.

\section{Conclusion}

In contrast to HVT, thiamine supplementation was associated with increased mortality rate in ICU. In addition, co-supplementation of thiamine and vitamin $\mathrm{C}$ had a significant decreasing effect on SOFA score.

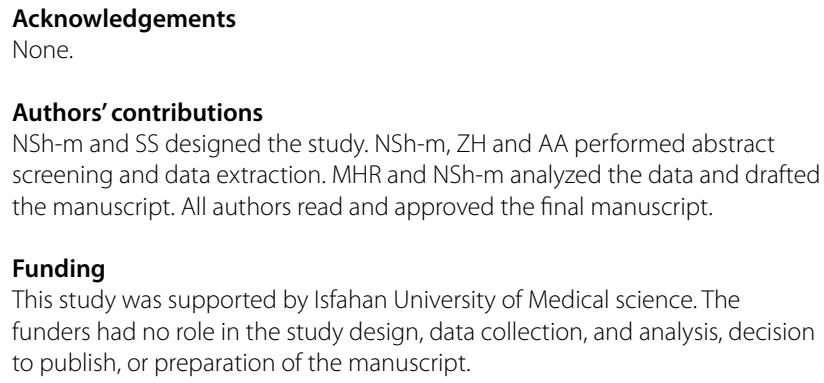

\section{Funding}

This study was supported by Isfahan University of Medical science. The funders had no role in the study design, data collection, and analysis, decision to publish, or preparation of the manuscript.

Availability of data and materials Not applicable.

\section{Declarations}

Ethics approval and consent to participate

Not applicable.

\section{Consent for publication}

The details/images/videos will be freely available on the internet and may be seen by the general public.

\section{Competing interests}

The authors declare that they have no competing interests.

\section{Author details}

${ }^{1}$ Food Security Research Center and Department of Clinical Nutrition, School of Nutrition and Food Science, Isfahan University of Medical Sciences, Isfahan, Iran. ${ }^{2}$ Food Security Research Center and Department of Community Nutrition, School of Nutrition and Food Science, Isfahan University of Medical Sciences, Isfahan, Iran. ${ }^{3}$ Faculty of Mathematics and Natural Sciences, Department of Computer Sciences, Heinrich Heine Universität, Düsseldorf, Germany.

Received: 11 May 2021 Accepted: 4 January 2022

Published online: 17 February 2022

\section{References}

1. Lonsdale D. A review of the biochemistry, metabolism and clinical benefits of thiamin (e) and its derivatives. Evid Based Comp Altern. 2006;3(1):49-59.

2. Van Stigt R, Van Der Wal G, Kamphuis S, Braber A. Beriberi in the ICU: Remarkable shock reversal with thiamine. Netherlands J Crit Care. 2019:27(2):93-6.

3. Baird JS, Ravindranath TM. Vitamin B deficiencies in a critically ill autistic child with a restricted diet. Nutr Clin Pract. 2015;30(1):100-3.

4. Ahmed HH, De Bels D, Attou R, Honore PM, Redant S. Elevated lactic acid during ketoacidosis: pathophysiology and management. JTIM. 2019;7(3):115-7.

5. Berger MM, Soguel L, Shenkin A, Revelly J-P, Pinget C, Baines M, et al. Influence of early antioxidant supplements on clinical evolution and organ function in critically ill cardiac surgery, major trauma, and subarachnoid hemorrhage patients. Crit Care. 2008;12(4):R101.

6. Moskowitz A, Graver A, Giberson T, Berg K, Liu X, Uber A, et al. The relationship between lactate and thiamine levels in patients with diabetic ketoacidosis. J Crit Care. 2014;29(1):182.e5-e8. 
7. Kim H, Stotts NA, Froelicher ES, Engler MM, Porter C. Why patients in critical care do not receive adequate enteral nutrition? A review of the literature. J Crit Care. 2012;27(6):702-13.

8. Rafiee S, Safari Z, Shokri-Mashhadi N. Current nutritional statuses and gastrointestinal complications in critically ill patients admitted to ICUs in Iran: a cross-sectional study. Nutr Food Sci Res. 2020;7(3):9-14.

9. Andersen LW, Holmberg MJ, Berg KM, Chase M, Cocchi MN, Sulmonte C, et al. Thiamine as an adjunctive therapy in cardiac surgery: a randomized, double-blind, placebo-controlled, phase II trial. Crit Care. 2016:20(1):1-11.

10. Moslemi R, Khalili H, Mohammadi M, Mehrabi Z, Mohebbi N. Thiamine for prevention of postoperative delirium in patients undergoing gastrointestinal surgery: a randomized clinical trial. J Res Pharm Pract. 2020;9(1):30.

11. Bedreag $\mathrm{OH}$, Rogobete $A F$, Sărăndan $M$, Cradigati $A C$, Păpurică $M$, Roşu $\mathrm{OM}$, et al. Influence of antioxidant therapy on the clinical status of multiple trauma patients. A retrospective single center study. Rom J Anaesth Intensive Care. 2015;22(2):89.

12. Harun NF, Cheah SK, Yusof AM, Lau CL, Masdar A, Mahdi SNM, et al. Intravenous thiamine as an adjuvant therapy for hyperlactatemia in septic shock patients. Crit Care Shock. 2019;22(6):288-98.

13. Lomivorotov V, Moroz G, Ismoilov S, Shmyrev V, Efremov S, Abubakirov $M$, et al. Sustained high-dose thiamine supplementation in high-risk cardiac patients undergoing cardiopulmonary bypass: a pilot feasibility study (the APPLY trial). J Cardiothorac Vasc Anesth. 2020;34(3):594-600.

14. Zayed Y, Alzghoul BN, Banifadel M, Venigandla H, Hyde R, Sutchu S, et al. Vitamin C, Thiamine, and corticostroid in the treatment of sepsis: a metaanalysis and trial sequential analysis of randomized controlled trials. J Intensive Care Med. 2021. https://doi.org/10.1177/0885066620987809.

15. Scholz SS, Borgstedt R, Ebeling N, Menzel LC, Jansen G, Rehberg S. Mortality in septic patients treated with vitamin C: a systematic meta-analysis. Crit Care. 2021;25(1):1-10.

16. Wu T, Hu C, Huang W, Xu Q, Hu B, Li J. Effect of combined hydrocortisone, ascorbic acid and thiamine for patients with sepsis and septic shock: a systematic review and meta-analysis. Shock. 2021. https://doi.org/10. 1097/SHK.0000000000001781.

17. Moskowitz A, Donnino MW. Thiamine (vitamin B1) in septic shock: a targeted therapy. J Thorac Dis. 2020;12(Suppl 1):S78-s83.

18. Moher D, Altman DG, Liberati A, Tetzlaff J. PRISMA statement. Epidemiology. $2011 ; 22(1): 128$

19. Jones AE, Trzeciak S, Kline JA. The Sequential Organ Failure Assessment score for predicting outcome in patients with severe sepsis and evidence of hypoperfusion at the time of emergency department presentation. Crit Care Med. 2009:37:1649-54.

20. Chan $\mathrm{CL}$, Ting HW, Huang HT. The definition of a prolonged intensive care unit stay for spontaneous intracerebral hemorrhage patients: an application with national health insurance research database. Biomed Res Int. 2014;2014:891725.

21. Yehya N, Harhay MO, Curley MAQ, Schoenfeld DA, Reeder RW. Reappraisal of ventilator-free days in critical care research. Am J Respir Crit Care Med. 2019;200:828-36.

22. Reitsma J, Rutjes A, Whiting P, Vlassov V, Leeflang M, Deeks J. Assessing methodological quality. Cochrane handbook for systematic reviews of diagnostic test accuracy version. 2009;1:1-28.

23. Iglesias J, Vassallo AV, Patel VV, Sullivan JB, Cavanaugh J, Elbaga Y. Outcomes of metabolic resuscitation using ascorbic acid, thiamine, and glucocorticoids in the early treatment of sepsis: the ORANGES trial. Chest. 2020;158(1):164-73.

24. Balakrishnan M, Gandhi H, Shah K, Pandya H, Patel R, Keshwani S, et al. Hydrocortisone, vitamin C and thiamine for the treatment of sepsis and septic shock following cardiac surgery. Indian J Anaesth. 2018;62(12):934.

25. Chang P, Liao Y, Guan J, Guo Y, Zhao M, Hu J, et al. Combined treatment with hydrocortisone, vitamin $C$, and thiamine for sepsis and septic shock: a randomized controlled trial. Chest. 2020;158(1):174-82.

26. Fujii T, Luethi N, Young PJ, Frei DR, Eastwood GM, French CJ, et al. Effect of Vitamin C, hydrocortisone, and thiamine vs hydrocortisone alone on time alive and free of vasopressor support among patients with septic shock: the VITAMINS randomized clinical trial. JAMA. 2020;323(5):423-31.

27. Hwang SY, Ryoo SM, Park JE, Jo YH, Jang D-H, Suh GJ, et al. Combination therapy of vitamin $C$ and thiamine for septic shock: a multi-centre, double-blinded randomized, controlled study. Intensive Care Med. 2020;46(11):2015-25.
28. Karimpour H, Bahrami A, Amini S, Rezaei M, Amini-Saman J, Shahbazi F. Effects of a high dose of vitamin C along with thiamine in critically-ill patients with septic shock: a preliminary study. JPRI. 2019. https://doi.org/ 10.9734/jpri/2019/v29i530248.

29. Moskowitz A, Huang DT, Hou PC, Gong J, Doshi PB, Grossestreuer $A V$, et al. Effect of ascorbic acid, corticosteroids, and thiamine on organ injury in septic shock: the ACTS randomized clinical trial. JAMA 2020;324(7):642-50

30. Wani SJ, Mufti SA, Jan RA, Shah S, Qadri SM, Khan UH, et al. Combination of vitamin C, thiamine and hydrocortisone added to standard treatment in the management of sepsis: results from an open label randomised controlled clinical trial and a review of the literature. Infect Dis. 2020;52(4):271-8

31. Donnino MW, Andersen LW, Chase M, Berg KM, Tidswell M, Giberson T, et al. Randomized, double-blind, placebo-controlled trial of thiamine as a metabolic resuscitator in septic shock: a pilot study. Crit Care Med. 2016;44(2):360.

32. Pradita-Ukrit $S$, Vattanavanit $\mathrm{V}$. Efficacy of thiamine in the treatment of postcardiac arrest patients: a randomized controlled study. Crit Care Res Practice. 2020. https://doi.org/10.1155/2020/2981079.

33. Luger M, Hiesmayr M, Köppel P, Sima B, Ranz I, Weiss C, et al. Influence of intravenous thiamine supplementation on blood lactate concentration prior to cardiac surgery: a double-blinded, randomised controlled pilot study. EJA. 2015;32(8):543-8.

34. Nasution AH, Yuli RS. The effect of thiamine administration on interleukin-6 (II-6) enzyme, lactate and sequential organ failure assessment (SOFA) score in patients with sepsis. J Drug Alcohol Res. 2020:9(4):1-5.

35. Dhir S, Tarasenko M, Napoli E, Giulivi C. Neurological, psychiatric, and biochemical aspects of thiamine deficiency in children and adults. Front Psych. 2019;10:207

36. Obi J, Pastores S, Ramanathan L, Yang J, Halpern N. Treating sepsis with vitamin C, thiamine, and hydrocortisone: exploring the quest for the magic elixir. J Crit Care. 2020;57:231-9.

37. Amrein K, Oudemans-van Straaten HM, Berger MM. Vitamin therapy in critically ill patients: focus on thiamine, vitamin C, and vitamin D. Intensive Care Med. 2018:44(11):1940-4.

38. Attaluri P, Castillo A, Edriss H, Nugent K. Thiamine deficiency: an important consideration in critically ill patients. Am J Med Sci. 2018;356(4):382-90.

39. Woolum JA, Abner EL, Kelly A, Bastin MLT, Morris PE, Flannery AH. Effect of thiamine administration on lactate clearance and mortality in patients with septic shock. Crit Care Med. 2018:46(11):1747-52.

40. Hernandez $G$, Bellomo R, Bakker J. The ten pitfalls of lactate clearance in sepsis. Intensive Care Med. 2019;45(1):82-5.

41. Finfer S, Machado FR. The global epidemiology of sepsis. Does it matter that we know so little? Am Thorac Soc. 2016;193:228-30.

42. Miyamoto $Y$, Aso S, Iwagami M, Yasunaga $H$, Matsui $H$, Fushimi $K$, et al. Association between IV thiamine and mortality in patients with septic shock: a nationwide observational study. Crit Care Med. 2020. https://doi. org/10.1097/CCM.0000000000004394

43. Collie JT, Greaves RF, Jones OA, Lam Q, Eastwood GM, Bellomo R. Vitamin B1 in critically ill patients: needs and challenges. Clin Chem Lab Med. 2017;55(11):1652-68.

44. Wilson JX. Mechanism of action of vitamin C in sepsis: ascorbate modulates redox signaling in endothelium. BioFactors. 2009;35(1):5-13.

45. Riess KP, Farnen JP, Lambert PJ, Mathiason MA, Kothari SN. Ascorbic acid deficiency in bariatric surgical population. Surg Obes Relat Dis. 2009;5(1):81-6.

46. Christen S, Finckh B, Lykkesfeldt J, Gessler P, Frese-Schaper M, Nielsen P, et al. Oxidative stress precedes peak systemic inflammatory response in pediatric patients undergoing cardiopulmonary bypass operation. Free Radical Biol Med. 2005;38(10):1323-32.

47. Kashiouris MG, L'Heureux M, Cable CA, Fisher BJ, Leichtle SW. The emerging role of vitamin C as a treatment for sepsis. Nutrients. 2020;12(2):292.

48. Lorente L, Martín MM, Almeida T, Abreu-González P, Ferreres J, Solé-Violán $J$, et al. Association between serum total antioxidant capacity and mortality in severe septic patients. J Crit Care. 2015;30(1):217.e7-e12.

49. Karakike E, Kyriazopoulou E, Tsangaris I, Routsi C, Vincent J-L, GiamarellosBourboulis EJ. The early change of SOFA score as a prognostic marker of 28-day sepsis mortality: analysis through a derivation and a validation cohort. Crit Care. 2019:23(1):387. 
50. Matsuda J, Kato S, Yano H, Nitta G, Kono T, Ikenouchi T, et al. The Sequential Organ Failure Assessment (SOFA) score predicts mortality and neurological outcome in patients with post-cardiac arrest syndrome. J Cardiol. 2020;76(3):295-302.

51. Soto ME, Guarner-Lans V, Soria-Castro E, Manzano Pech L, Pérez-Torres I. Is antioxidant therapy a useful complementary measure for Covid-19 treatment? An algorithm for its application. Medicina. 2020;56(8):386.

52. Wei X-B, Wang Z-H, Liao X-L, Guo W-X, Wen J-Y, Qin T-H, et al. Efficacy of vitamin $C$ in patients with sepsis: an updated meta-analysis. Eur J Pharmacol. 2020;5:868.

53. Jonckheer J, Vergaelen K, Spapen H, Malbrain ML, De Waele E. Modification of nutrition therapy during continuous renal replacement therapy in critically ill pediatric patients: a narrative review and recommendations. Nutr Clin Pract. 2019;34(1):37-47.

54. Ince C, Mayeux PR, Nguyen T, Gomez H, Kellum JA, Ospina-Tascón GA, et al. The endothelium in sepsis. Shock (Augusta, GA). 2016;45(3):259.

55. Marik PE. Vitamin C for the treatment of sepsis: the scientific rationale. Pharmacol Ther. 2018;189:63-70.

56. Moreno R, Sprung C, Annane D, Chevret S, Briegel J, Keh D, et al. Time course of organ failure in patients with septic shock treated with hydrocortisone: results of the Corticus study. In: Pinsky MR, Brochard L, Hedenstierna G, Antonelli M, editors., et al., Applied physiology in intensive care medicine 1. Berlin: Springer; 2012. p. 423-30.

57. Marik PE. Hydrocortisone, ascorbic acid and thiamine (HAT therapy) for the treatment of sepsis. Focus on ascorbic acid. Nutrients. 2018;10(11):1762.

58. Shi R, Tie H. Benefit of hydrocortisone, thiamine, and vitamin C for patients with sepsis or septic shock? Too early to draw conclusions. Crit Care. 2020;24(1):1-5.

59. Rygård SL, Butler E, Granholm A, Møller MH, Cohen J, Finfer S, et al. Lowdose corticosteroids for adult patients with septic shock: a systematic review with meta-analysis and trial sequential analysis. Intensive Care Med. 2018;44(7):1003-16.

60. Cabbab ILN, Manalo RVM. Anti-inflammatory drugs and the renin-angiotensin-aldosterone system: current knowledge and potential effects on early SARS-CoV-2 infection. Virus Res. 2021;291:198190.

61. Ivanov V, Goc A, Ivanova S, Niedzwiecki A, Rath M. Inhibition of ACE2 expression by ascorbic acid alone and its combinations with other natural compounds. Infect Dis. 2021. https://doi.org/10.1177/1178633721 994605.

62. Xiang Z, Liu J, Shi D, Chen W, Li J, Yan R, et al. Glucocorticoids improve severe or critical COVID-19 by activating ACE2 and reducing IL-6 levels. Int J Biol Sci. 2020;16(13):2382-91.

63. Yasir M, Goyal A, Bansal P, Sonthalia S. Corticosteroid adverse effects. In: StatPearls [Internet]. Treasure Island (FL): StatPearls Publishing; 2022.

64. Jordan Parker MD, Yuan S, Donohue M, Brown R, Sutherland M, Goldberg $\mathrm{H}$, Honasoge A. Do adjunctive therapies beyond infection control and appropriate fluid resuscitation change outcomes in sepsis and septic shock? Chest. 2017;151(6):1229-38.

65. Counts JP, Rivera VF, Kimmons LA, Jones GM. Thiamine use in sepsis: B: 1 : for everyone? Crit Care Nurs Q. 2019;42(3):292-303.

66. Wei XB, Wang ZH, Liao XL, Guo WX, Wen JY, Qin TH, et al. Efficacy of vitamin C in patients with sepsis: an updated meta-analysis. Eur J Pharmacol. 2020;868:172889.

67. Somagutta MKR, Pormento MKL, Khan MA, Hamdan A, Hange N, Kc M, et al. The Efficacy of vitamin C, thiamine, and corticosteroid therapy in adult sepsis patients: a systematic review and meta-analysis. Acute Crit Care. 2021. https://doi.org/10.4266/acc.2021.00108.

68. Ge Z, Huang J, Liu Y, Xiang J, Gao Y, Walline JH, et al. Thiamine combined with vitamin $\mathrm{C}$ in sepsis or septic shock: a systematic review and metaanalysis. Eur J Emerg Med. 2021;28(3):189-95.

\section{Publisher's Note}

Springer Nature remains neutral with regard to jurisdictional claims in published maps and institutional affiliations.

Ready to submit your research? Choose BMC and benefit from:

- fast, convenient online submission

- thorough peer review by experienced researchers in your field

- rapid publication on acceptance

- support for research data, including large and complex data types

- gold Open Access which fosters wider collaboration and increased citations

- maximum visibility for your research: over 100M website views per year

At BMC, research is always in progress.

Learn more biomedcentral.com/submissions 